\title{
Fat
}

\section{Rapporteur's report}

\section{Chair: Kaare R. Norum ${ }^{\prime}$}

Rapporteur: Tim Lobstein ${ }^{\star 2}$

1 University of Oslo, P.O. Box 1072 Blindern, N-0316 Oslo, Nonway

2 International Association of Consumer Food Organisations (IACFO) and the Food Commission,

94 White Lion St., London N1 9PF, UK

*Correspondence: Email foodcom @ compuserve.com

\section{Presentations}

\section{Obesity and Diabetes}

Ame Astrup, The Royal Veterinary and Agricultural University, Denmark

Dietary Alpha-Linolenic Acid for Prevention of Cardiovascular Diseases

Serge Renaud et al, INSERM, France

Derivation of food-based dietary guidelines. Examples for dietary fat using dietary survey data

Wulf Becker, Swedish National Food Administration, Sweden

Kaare R. Norum introduced the session by reminding participants that recommendations for reducing fat consumption in the general diet had been made around 40 years ago by expert committees in Norway, which had specified both a reduction in total fat consumption and a reduction in saturated fat consumption, and had also recommended an increase in the ratio of polyunsaturated fats to saturated fats. These recommendations had been accepted as official policy for at least 25 years.

Arne Astrup presented an overview of the evidence linking obesity with diabetes type II, and suggested that even modest weight loss - say of $3 \mathrm{~kg}$ - among the obese population would reduce the incidence of diabetes type II by as much as $60 \%$ in this group. The key factor is weight, and this is supported by data from studies of the outcome of people experiencing gastric surgery - weight loss surgery for obesity reduces the risk of diabetes significantly.

To reduce the incidence of obesity in the general population by a significant amount, while minimising the likelihood of encouraging underweight, an average BMI of 22 is recommended. Energy balance is best achieved with diets containing $20-25 \%$ energy from fat for sedentary subjects, and $25-30 \%$ fat for highly physically active subjects.

It should be noted that exercise is an independent and important co-factor in reducing the incidence of diabetes type II. Also, as with a reduction in obesity, exercise can have a marked impact on reducing blood pressure, the risk of stroke and myocardial infarction rates.

Serge Renaud discussed several reports on the role of different fatty acids and the results that can be achieved when changing the fatty acid profile of the diets of coronary patients. Remarkable improvements were observed when coronary patients were put on a Cretan-style diet containing the following components:

$8 \%$ saturated fat $(12 \%$ for controls)

$5 \%$ polyunsaturated fat, including $2 \mathrm{~g}$ alpha linolenic more cereal and vegetables

fish twice per week

cheese, yogurt, fruit

Within a follow up period of around two years, three deaths had occurred among nearly 300 patients, compared with 16 deaths among a similar sized control group. The effects were sufficiently dramatic to bring the experiment to a halt, allowing the control group to adopt a healthier diet. Cancer rates also showed some improvement, with less than half the rates in the experimental group over a four-year period.

Wulf Becker showed a map of the average fat intakes ranging from $43 \%$ down to $31 \%$ for countries and/or sub-populations of western Europe, based on the Transfair study. ${ }^{1}$ A simple North-South pattern was not applicable - Nordic 
countries had a rate ranging from $31-42 \%$ of energy from fat and the Mediterranean from 30 $41 \%$. Becker suggested that simple advice for the public to reduce fat consumption was not as helpful as advice giving recommendations on the types and amounts of fat and oil that should be consumed. Advice should also be given on what to replace the fat with: sweet food or alcohol was not suitable while complex carbohydrates, fruit and cereals were. Advice should be offered to the general population and also specifically to caterers, and there was a need to improve the quality of processed foods and foods from take-aways and restaurants.

In the discussions, it was clear that different advice was needed for different cultural circumstances: e.g. high olive oil consumers might want different dietary advice compared with consumers of high levels of meat and dairy fats. Even the word 'fat' can be misinterpreted by the public - and by policy makers - and the word 'lipids', or the phrase 'fats and oils', would be preferable.

For population averages, the group considered Table 1 of Working Group 1, and suggested that a range of population goals of $25 \%-35 \%$ energy from fat was consistent with good health, with an overall average less than $30 \%$. For physically inactive populations a lower figure is preferred. Averages above $30 \%$ should be associated with increased physical activity. An advantage of lower fat consumption is a generally improved intake of micronutrient levels.

For saturated fats, a population average of not more than $10 \%$ energy was agreed, and not more than $2 \%$ from trans fats (and this could be included within the saturated fat figure).

There was some discussion about the need for a monounsaturated fat figure, and this was left undetermined, although a lower limit of $10 \%$ energy was suggested.

For polyunsaturated fats, proposals varied a little, with general agreement around the levels of $5 \%$ energy from $n-6$ and $1 \%$ energy from $n-3$, and a minimum of 1 gram of $n-3$.

Lastly, concern about the process of arriving at figures was expressed, with fears that we were basing ourselves on eminence-based science rather than evidence-based science. The group agreed to look into setting up a Cochrane review panel for the evidence on the role of dietary fats, which might better allow the conclusions to be based on agreed methods of data assessment. Eric Brunner (e.brunner@ucl. ac.uk) offered to act as an initial coordinator.

\section{Reference}

1. Hulshof KFAM, van Erp-Baart MA, Anttolainen M, Becker W, Church SM, Couet C et al. Intake of fatty acids in Westem Europe with emphasis on trans fatty acids: The TRANSFAIR study. Eur J Clin Nutr 1999; 53: 143-57.

\section{Abstracts}

\section{Obesity and diabetes}

Arne Astrup*

Research Department of Human Nutrition, The Royal Veterinary and Agricultural University, Rolighedsvej 30,

1958 Frederiksberg C, Denmark

*Correspondence: Email ast@kvl.dk

Keywords

obesity, diabetes, dietary fat, energy balance, physical activity

The prevalence of obesity is increasing rapidly in all age groups in most EU-countries and is one of the fastest growing epidemics, now affecting 10-20 $\%$ of the adult population. Obesity is followed by serious co-morbidities such as type 2 diabetes, cardiovascular disease, certain cancers and reduced life expectancy, and these complications may account for $5-10 \%$ of all health costs in EU countries. It is particularly the risk of diabetes that is increased by obesity, up to 100 -fold, and at least $80 \%$ of the increasing number of diabetics can be attributed to obesity and overweight with abdominal fat 
distribution. There is robust evidence by crosssectional and longitudinal studies to support that an energy-dense, high fat diet and physical inactivity are independent risk factors for weight gain and obesity. Furthermore, interactions between dietary fat and physical fitness determine fat balance, so that the obesity promoting effect of a high fat diet is enhanced in susceptible subjects, particularly in sedentary individuals with a genetic predisposition to obesity. A diet with a higher fat content seems to be better tolerated without weight gain by physically active individuals than by sedentary people.

Ad libitum consumption of diets low in fat and high in protein and complex carbohydrates, with a low glycemic index, contributes to the prevention of weight gain in normal weight subjects. It also causes a spontaneous weight loss of $3-4 \mathrm{~kg}$ in overweight subjects, and has beneficial effects on risk factors for diabetes and CVD. The addition of daily physical activity to the diet doubles the weight loss in overweight subjects. Implementation of this dietary change and increased physical activity may reduce the mean body weight of the population and decrease the prevalence of obesity and diabetes substantially. In conclusion, important interactions exist between genetic make up, dietary fat and physical fitness, and increasing daily physical activity and reducing dietary fat content may be more effective in combination than separately in preventing weight gain and obesity. Energy balance seems only to be achieved with less energy dense diets and fat intakes of $20-25$ energy \% in sedentary subjects, but $25-30 \%$ in the highly physically active.

\section{Dietary alpha-linolenic acid for prevention of cardiovascular diseases}

\section{S. Renaud ${ }^{\star 1}$, D. Lanzmann-Petithory ${ }^{2}$}

1INSERM, Unité 330, Université Bordeaux 2, 146 rue Léo Saignat, 33076 Bordeaux Cedex, France

2Hôpital Emile Roux, Pavillon Buisson-Jacob, Service de Geriatrie, 1 Avenue Verdun, 94456 Limeil-Prevannes, Cedex, France

${ }^{\star}$ Correspondence: Email : Serge.Renaud @ bordeaux.inserm.fr

Keywords

Alpha linolenic acid, cardiac death, thrombosis, Cretan diet

The intake of saturated fat was postulated to be the main environmental factor for coronary heart disease. It was also believed that the noxious effects of saturated fatty acids was primarily through the increase in serum cholesterol. Nevertheless intervention trials either in coronary patients or even in primary prevention did not observe significant reduction in cardiac mortality, especially sudden death, when the diet was markedly enriched in linoleic acid, the main fatty acid that lowers serum cholesterol. It is only when the diet was enriched in $\mathbf{n}-3$ fatty acids, by higher fish intake or fish oil that cardiac death was reduced. Studies in animals as well as in vitro, on myocytes in culture, have shown that $n-3$ fatty acids were preventing ventricular fibrillation, the chief mechanism of cardiac death. Furthermore, studies in rats have observed that among $\mathrm{n}-3$ fatty acids, alpha-linolenic acid (ALA), the precursor of the $n-3$ family, may be more efficient to prevent ventricular fibrillation than EPA and DHA. In addition we had previously demonstrated that ALA was the main fatty acid lowering platelet aggregation an important step in coronary thrombosis. The two populations documented to have the greatest life expectancy, the Japanese in Japan and the Cretans in Crete have a high plasma level of ALA. To evaluate to what extent the diet could explain the Cretan longevity, in a randomized trial on 600 coronary patients in Lyon ${ }^{1}$, we compared an adaptation of the Cretan diet to the prudent diet commonly prescribed. After a mean follow-up period of 27 months all cardiovascular events, cardiac and total death were significantly $(\mathrm{p}<0.001)$ decreased by more than $70 \%$ in the group with the Cretan diet. These protective effects on clinical manifestations of $\mathrm{CHD}$ were not related to total or $\mathrm{HDL}$ cholesterol, Apoproteins $\mathrm{A}$ or $\mathrm{B}$, triglycerides or Lipoprotein (a) in serum.

By contrast, the protective effects were associated with an increase in plasma of alpha-linolenic and oleic acids, and a decrease in linoleic acid and saturated fatty acids. That protection was already observed after 2 months of diet and was probably due to an inhibitory effect of thrombosis via platelets and of sudden death, via ventricular fibrillation.

Recent prospective studies in women ${ }^{2}$ in USA (Harvard Public Health) indicate that the only fatty acid apparently 
inhibiting cardiac mortality in man was alphalinolenic acid. Thus, alpha-linolenic acid, in addition to regulate the level of prostaglandins and leukotrienes, may be the main fatty acid protecting from CHD clinical manifestations, especially sudden death.

In addition, since further evaluation on the effects of ALA in our Lyon diet heart study has shown after 4 years of follow up ${ }^{3}$ that cancer was reduced by $58 \%$ in the group with a high intake of ALA, this fatty acid may be much more important for health than previously suspected.

\section{References:}

1. Renaud S., de Lorgeril M., Delaye J. et al. Cretan Mediterranean diet for prevention of coronary heart disease. Am J Clim Nutr, 1995;61(Suppl):1360S-1367S.

2. De Lorgeril M., Salen P., Martin J.L., Monjaud I., Boucher P., Mamelle N. Mediterranean dietary pattern in a randomized trial. Prolonged survival and possible reduced cancer rate. Arch Intern Med, 1998;158:1181-7.

3. Hu FB, Stampfer MJ, Morison JE et al. Dietary intake of alpha-linolenic acid and risk of fatal ischaemic heart disease among women. Am J Clin Nutr 1999;69:890-7.

\section{Derivation of food-based dietary guidelines. Examples for dietary fat using dietary survey data}

Wulf Becker*

Nutrition Division, Swedish National Food Administration, Uppsala, Sweden

*Correspondence: Email wube @slv.se

Keywords

Food-based dietary guidelines, fat intake, fat quality, dietary

surveys

Objectives. To study food patterns explaining variations in fat intake in European populations and the implications for dietary guidelines.

Methods. Dietary data for Austria, Belgium, Finland, Germany, Greece, Ireland, Italy, Netherlands, Portugal, Spain, Sweden, and United Kingdom were used to identify food patterns among low and high consumers of fat.

Results. The data show, with a few exceptions, that a low intake of both total and saturated fat is associated with a high consumption of fruit, less frequently so to consumption of vegetables, and to a lower intake of cheese and spreads. Other typical characteristics of a low fat diet are high consumption of cereal foods (whole grain) and sometimes a high intake of simple sugars and alcohol. Sex differences were seen, e.g. for cheese and alcoholic beverages. Since the fat intake is expressed in relative terms, consumption of low-fat foods such as cereals, sugar-rich foods and alcoholic beverages also contribute to the variation in energy $\%$ fat. Fat composition was virtually the same among low and high consumers of fat.

Conclusions. These data show that there are some common characteristics of low and high fat diets in Europe, which could be used as a basis for some general dietary guidelines. For certain foods trends were divergent between countries. More detailed and targeted guidelines have to be based on the dietary pattern of the individual country or population. 